Mejoramiento de la Calidad de vida de los beneficiarios del Programa de Pensión Alimentaria para Adultos Mayores en situación de pobreza en la Colonia Indígena La Promesa en el Departamento de Presidente Hayes ${ }^{1}$

\title{
Improvement of the quality of life of the beneficiaries of the Alimony Pension Program for the Elderly in a situation of poverty in the La Promesa Indigenous Colony in Presidente Hayes
}

\section{Santiago Lorenzo Galeano Bate ${ }^{2}$}

\author{
${ }^{2}$ Universidad Columbia del Paraguay. Asunción, Paraguay. \\ Recibido: 28/04/2018 Aceptado: 05/06/2018
}

\section{RESUMEN}

Según la Ley N³728/09 todo paraguayo natural, mayor de 65 años de edad y en situación de pobreza, residente en el territorio nacional, recibirá del Estado una pensión mensual no menor a la cuarta parte del salario mínimo vigente. El objetivo general es la de Conocer el mejoramiento de la Calidad de vida de los beneficiarios del Programa de Pensión Alimentaria para Adultos Mayores en situación de pobreza en la Colonia Indígena La Promesa en el Departamento de Presidente Hayes, constituye una información relevante para medir el impacto de este tipo de programas en comunidades indígenas aisladas. La investigación es del tipo cualitativa no experimental descriptiva, utiliza la modalidad bibliográfica documental y de campo, con los métodos de entrevista y registros de observación. Los resultados obtenidos presentan las características de los beneficiarios del Programa de Pensión Alimentaria para Adultos Mayores en situación de pobreza, así como aspectos relacionados al mejoramiento de la calidad de vida de los beneficiarios del Programa.

Palabras clave: Adultos mayores, Pensión, Pueblos Originarios

\section{ABSTRACT}

According to Law No. 3728/09, all natural Paraguayan, over 65 years of age and living in poverty, residing in the national territory, will receive from the State a monthly pension not less than a quarter of the current minimum wage. The general objective of the program is to know the improvement of the quality of life of the beneficiaries of the Alimony Pension Program for the elderly living in poverty in the Indigenous Colony La Promesa in the Department of Presidente

${ }^{1}$ Trabajo de Investigación de la Facultad de Ciencias Económicas (FCE), financiado por el Rectorado de la Universidad Nacional de Asunción en el 2016. 
Hayes is a relevant information to measure the impact of this type of programs in isolated indigenous communities. The research is of the qualitative non-experimental descriptive type, it uses the documentary and field bibliographic modality, with the interview methods and observation records. The results obtained present the characteristics of the beneficiaries of the Alimony Pension Program for the Elderly in a situation of poverty, as well as aspects related to the improvement of the quality of life of the beneficiaries of the Program.

Key words: Older adults, Pension, Original towns

Autor correspondiente: Santiago Lorenzo Galeano Bate. Licenciado en Administración. Docente de tiempo completo de la carrera de Hotelería y Turismo. Universidad Columbia del Paraguay. Asunción, Paraguay. Email: sagabas@gmail.com

\section{INTRODUCCIÓN}

La colonia La Promesa es una de las 46 comunidades indígenas ubicadas dentro del Departamento de Presidente Hayes, actualmente cuenta con 270 habitantes. Dentro del contexto actual, hoy en día 12 adultos mayores de dicha comunidad cobran la pensión alimentaria para adultos mayores en situación de pobreza, desde el 2012.

De acuerdo a la Ley N³728/09 todo paraguayo natural, mayor de sesenta y cinco años de edad y en situación de pobreza, residente en el territorio nacional, recibirá del Estado una pensión mensual no menor a la cuarta parte del salario mínimo vigente.

Esta ley obedece a que en los últimos años el país ha iniciado un proceso de envejecimiento de su población debido principalmente al incremento en la expectativa de vida y a un descenso en el nivel de mortalidad y tasa de natalidad de su población, además del escaso acceso de las personas adultas mayores a la seguridad social.

En la actualidad no se conocen de manera particular los resultados de la implementación de la Ley $N^{\circ} 3728 / 09$ en la comunidad indígena La Promesa.

De acuerdo a la Constitución Nacional de la República del Paraguay, promulgada en el año 1992 en su artículo 6 expresa que "la calidad de vida será promovida por el Estado mediante planes y políticas que reconozcan factores condicionantes, tales como la extrema pobreza y los impedimentos de la discapacidad o de la edad".

Además, en el artículo 57 menciona que "toda persona de la tercera edad tiene derecho a una protección integral. La familia, la sociedad y los poderes públicos promoverán su bienestar mediante servicios sociales que se ocupen de sus necesidades de alimentación, salud, vivienda, cultura y ocio".

En el 2009 el Congreso de la Nación Paraguaya sanciona la Ley N³728, que establece el derecho de pensión alimentaria para personas adultas mayores en situación de pobreza. El ministerio de Hacienda comenzó a pagar a los primeros 909 adultos mayores en el año 2010. A mayo de 2016, el subsidio alcanza a 151.311 adultos mayores en todo el país de acuerdo a datos de la Dirección de Pensiones No Contributivas (DPNC) del Ministerio de Hacienda.

Los regímenes de pensiones no contributivos abarcan una serie de transferencias públicas destinadas a las personas mayores pobres y a sus hogares (Barrientos, 2003).

En el país estos regímenes no están sujetos a comprobaciones de la inactividad económica, además no se cuentan con resultados del impacto que los mismos generan en la población de destino. 
Los planes de reducción de la pobreza basados en pensiones no contributivas se están extendiendo rápidamente en los países en desarrollo y constituyen una nueva fuente de asistencia para la vejez. (Barrientos, 2007).

En la región se tienen los siguientes ejemplos de este tipo de subsidio; Bonosol o Renta de Dignidad en Bolivia, el programa de oportunidades en México, el Bono Desarrollo Humano en Ecuador o el programa Juntos en Perú.

Según Zavattiero (2010) en el país este tipo de pensión no contributiva logra reducir el porcentaje de pobreza en cierto grado, no obstante, sería necesario replantear la legislación a modo de aumentar la eficiencia de la medida.

De acuerdo a datos del Tercer Censo Nacional de Población y Viviendas para Pueblos Indígenas, el 76 por ciento de los aproximadamente 116.000 nativos del territorio de Paraguay se encuentran en situación de pobreza extrema.

En la comunidad indígena La Promesa existen 12 beneficiarios del Programa de Pensión Alimentaria para Adultos Mayores, teniendo en cuenta esta situación ¿̇Eiste un mejoramiento en la calidad de vida de los adultos mayores de la comunidad Indígena La Promesa, luego de la inclusión de los mismos dentro del Programa de Pensión Alimentaria para Adultos Mayores?

Esta información relacionada al impacto que puede generar este tipo de pensión en comunidades indígenas y rurales aisladas no existe actualmente. Este manuscrito busca presentar las características de los beneficiarios del Programa de Pensión Alimentaria para Adultos Mayores en situación de pobreza en la colonia Indígena La Promesa.

\section{MATERIALES Y MÉTODOS}

La presente investigación utiliza el enfoque cualitativo, donde el diseño de la investigación es del tipo no experimental, transversal, descriptivo. Donde primeramente se utilizó la modalidad bibliográfica documental, para evaluar las fuentes de información coherentes con los objetivos de la investigación.

Posteriormente se realizó una investigación por encuesta de percepción, donde el instrumento utilizado fue la entrevista, además se realizaron registros de observación para complementar los datos recabados en las entrevistas.

La población estuvo conformada por todos los adultos mayores beneficiarios del programa, de la comunidad indígena La Promesa.

Al ser una población pequeña se tomó la totalidad de la misma como muestra, incluyendo además una población de control, que estuvo constituida por los adultos mayores de la comunidad que no son beneficiarios del Programa de Pensión Alimentaria para Adultos Mayores en situación de pobreza (Tabla 1).

Tabla 1: Adultos mayores de la comunidad Indígena La Promesa. Año 2016.

\section{Población}

Cantidad

Adultos mayores beneficiarios del programa

Adultos mayores no beneficiarios

Total de adultos mayores de la comunidad indigena La Promesa

Fuente: Elaboración propia. Investigación de campo. Encuesta realizada el 27/08/16. 
La población de control está constituida por 5 adultos mayores que no son beneficiarios del programa debido a que no cuentan con cedula de identidad policial, uno de los requisitos para poder acceder a la pensión.

La población de control sirvió para comparar con la muestra, si existe un mejoramiento en las condiciones de vida de los beneficiarios del programa, al aplicarse el mismo cuestionario para ambos grupos.

El cuestionario utilizado para los adultos mayores beneficiarios y no beneficiarios del programa estuvo conformado por 10 preguntas abiertas, todas ellas referentes a conocer el mejoramiento de la calidad de vida de los beneficiarios del Programa de Pensión Alimentaria para Adultos Mayores en situación de pobreza.

La administración del instrumento se realizó de forma individual y se logró la aceptación y participación activa de los actores involucrados en la investigación gracias a la facilitación y presencia del líder comunitario (cacique) local Benito Fleitas.

Los principales inconvenientes encontrados al momento de la investigación fueron, las condiciones de los caminos de acceso a la comunidad, que impidió el trabajo en los días de lluvia, debido a las condiciones de los mismos. Se utilizaron medios para la conservación y archivo de todos los datos obtenidos durante la investigación de campo.

\section{RESULTADOS Y DISCUSIÓN}

Los adultos mayores de la colonia Indígena La Promesa, habitan en la misma desde su fecha de fundación el 29 de setiembre de 1987. Antes de la fecha de fundación los actuales pobladores vivían en tolderías dentro del predio de dos estancias vecinas al lugar; Estancia La Pastora y Estancia Cerrito.

Actualmente la colonia Indígena cuenta con 270 habitantes, distribuidos en 45 viviendas, donde la población de adultos mayores es de 17 personas, según datos aportados por el líder comunitario Benito Fleitas.

Los beneficiarios del Programa de Pensión Alimentaria para Adultos Mayores en situación de pobreza son 12 personas en total en la colonia La Promesa, de las cuales 2 adultos mayores son marido y mujer, por lo tanto, son 11 unidades familiares las beneficiadas con la "presencia" de al menos un adulto mayor dentro del hogar. Lo que según ellos constituye una gran ventaja, ya que de esta forma cuentan con ingresos permanentes que les permite acceder a la compra de alimentos de manera continua.

Opiniones realizadas por algunos de los entrevistados lleva a entender que muchos pobladores que aún no acceden a la pensión por no tener la edad mínima, esperan con mucha expectativa y esperanza, llegar a la edad establecida para así contar con un ingreso seguro que les permita cubrir sus necesidades de alimentación.

Dentro de este contexto, de las 45 viviendas existentes en la colonia Indígena La Promesa, 11 viviendas reciben el subsidio correspondiente a adultos mayores en situación de pobreza (figura 1). 
Figura 1. Viviendas existentes en la colonia Indígena La Promesa.

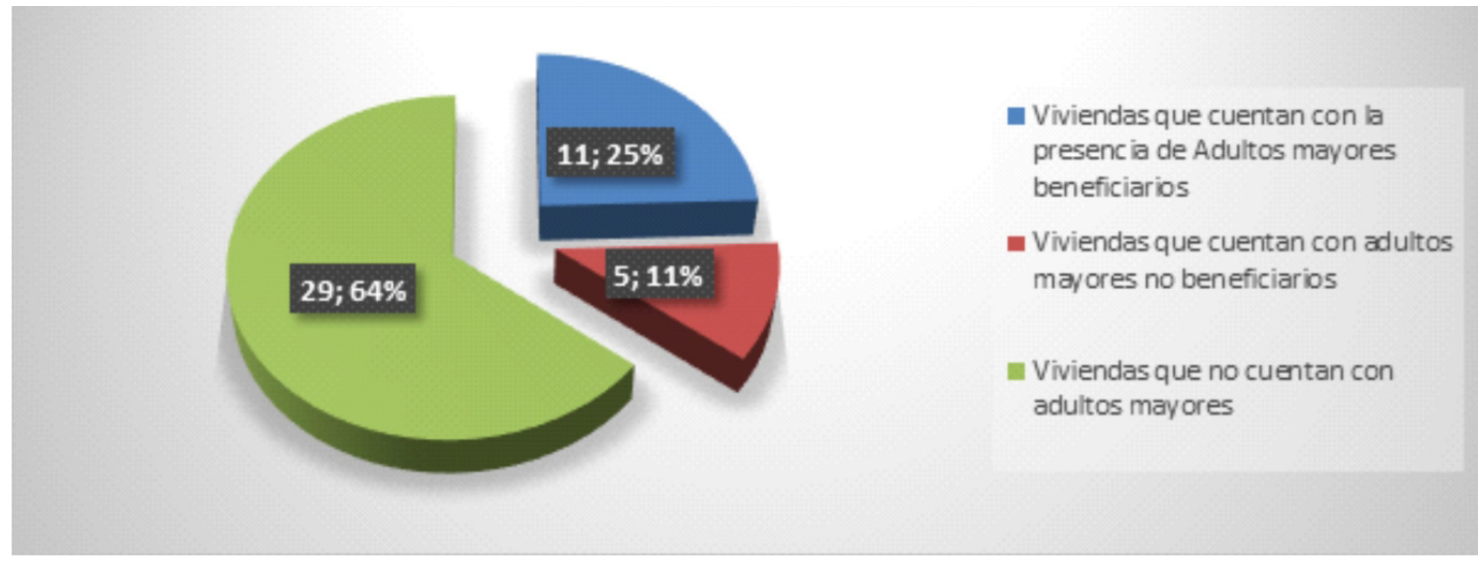

Fuente: Elaboración propia. Investigación de campo. Encuesta realizada el 27/08/16.

Los beneficiarios del Programa de Pensión Alimentaria para Adultos Mayores de la colonia Indígena La Promesa, en un 100\% habitan en viviendas tipo rancho, que están hechas de estaqueo de caranday (palmas), piso de tierra y techo de teja de caranday.

La colonia Indígena La promesa, no cuenta con servicio de energía eléctrica provisto por la Ande. Todas las viviendas cuentan con servicio sanitario a partir de letrina común, realizadas mediante la donación de una Organización No Gubernamental, en el 2012.

Las viviendas no cuentan con bañaderas particulares, la comunidad tiene 5 piezas de baño a disposición de todos los pobladores, obra que también fue realizada por una Organización No Gubernamental, según datos brindados por el líder comunitario.

El agua que se utiliza para beber se obtiene de los tajamares existentes en la colonia y en épocas de sequía los pobladores se trasladan a territorios cercanos para conseguir el vital líquido, también de tajamares. En días de lluvia colectan el agua en tambores o bidones.

En relación a la cantidad de personas que habitan en la vivienda donde reside el beneficiario de la pensión, la pregunta fue la siguiente ¿Cuántas personas habitan en la vivienda? En relación a la cantidad total de personas en el hogar, los porcentajes mayores tienen entre seis y siete personas habitando en la misma vivienda, del beneficiario del programa. Lo que indica que el ingreso proveniente de la pensión del adulto mayor beneficia de manera indirecta a cuatro personas o más.

En relación conocer si alguna persona que vive en el hogar realiza algún tipo de actividad remunerada de manera permanente u ocasional la pregunta planteada a los entrevistados fue la siguiente ¿Existe en el hogar alguna persona que realiza una actividad remunerada?

De las once viviendas que cuentan con adultos mayores beneficiarios del programa, solamente dos de ellas cuentan con personas que realizan algún tipo de actividad remunerada. Estas actividades tienen que ver principalmente con trabajos o changas en estancias vecinas, con una frecuencia de trabajo de 4 a 5 días al mes, con un pago diario.

Las condiciones de aislamiento debido al mal estado de los caminos que llevan a la comunidad, impide que los pobladores puedan trasladarse a sitios más lejanos para trabajar, donde podrían encontrar mejores condiciones de ingreso.

Teniendo en cuenta la condición de aislamiento de la colonia se realizó la siguiente pregunta; ¿De qué manera realiza el cobro de la pensión?, las respuestas se observan en la tabla 2. 
Tabla 2. Forma de cobro de la pensión. Año 2016.

\begin{tabular}{lc}
\hline Población & Cantidad \\
\hline De manera personal ( a través de cajeros automáticos) & 1 \\
Por medio de terceros (maceteros) & 11 \\
$\begin{array}{l}\text { Total de adultos mayores beneficiarios del programa de la comunidad } \\
\text { indígena La Promesa }\end{array}$ \\
\hline
\end{tabular}

Fuente: Elaboración propia. Investigación de campo. Encuesta realizada el 27/08/16.

Todos los beneficiarios de la pensión cobran a través de la red bancaria Dinelco por medio de cajeros automáticos, lo que de alguna forma dificulta el cobro de los beneficiarios en la colonia. En la actualidad el monto de la pensión pagada alcanza 456.014GS. por cada beneficiario, lo que equivale al $25 \%$ del salario mínimo vigente.

De acuerdo a los datos, 11 de los 12 beneficiarios entregan sus tarjetas de débito a "macateros" quienes se encargan de retirar el dinero. En algunos casos, el dinero en su totalidad o en parte no les es entregado ya que los "macateros" directamente les entregan mercaderías por el valor de la pensión.

El cobro de la pensión se realiza de manera mensual, el inconveniente presentado en algunos meses es el acceso a la colonia, que imposibilita el cobro de la pensión, debido a las malas condiciones del camino.

A los efectos de conocer a que destina el dinero recibido de la pensión se planteó la siguiente pregunta ¿A que dedica el dinero del subsidio? Donde el $100 \%$ de los encuestados menciono que el primer gasto en relación al dinero del subsidio va para compra de alimentos y en segundo lugar para compra de ropa. Esta situación se verifica en función a la respuesta anterior donde los macateros son los que cobran de manera efectiva el subsidio, ya que posteriormente entregan a los beneficiarios provistas (alimentos) o ropas.

En relación al nivel educativo ninguno de los beneficiarios cuenta con algún tipo de formación, es decir no saben leer ni escribir.

Consultados en relación a saber si tienen algún tipo de enfermedad, de los doce encuestados 8 mencionaron que padecen algún tipo enfermedad o dolencia y deben seguir algún tipo de tratamiento.

En relación a lo anterior se presenta lo siguiente ¿Dónde realiza su tratamiento médico? Debido a las condiciones del camino, lo que dificulta la rápida entrada y salida a la comunidad, y los costos de traslado hasta la ciudad de Villa Hayes distante a $200 \mathrm{~km}$. de la colonia o Pozo Colorado distante a 90 km., los adultos mayores siguen sus tratamientos o consultas médicas en el Puesto de Salud de la colonia, que se encuentra a cargo de un Licenciado en Enfermería.

El Puesto de Salud de la colonia, cuenta con una infraestructura mínima, como para poder ofrecer una buena atención en salud. En muchos casos los macateros son los encargados de traer los medicamentos necesarios que luego son vendidos a los beneficiarios de la pensión que lo necesiten.

Otra pregunta dirigida a los beneficiarios fue la siguiente ¿En los últimos 90 días realizó algún tipo de trabajo remunerado? Donde las respuestas fueron: 
Solamente 3 de los beneficiarios realizaron algún tipo de trabajo o actividad remunerada en los últimos 90 días. Las tres corresponden a personas del sexo masculino. Donde la actividad consistió en realizar trabajo de estancia, donde el pago fue por día de trabajo y con una frecuencia de 5 días al mes.

Esta situación fue comentada por los beneficiarios indicando que hoy en día existe una necesidad de personal de campo a caballo en las estancias vecinas. Que es cubierta en cierta medida por algunos adultos mayores que residen en la colonia, que son "baqueanos" o experimentados en dicha actividad. Mencionan además que los jóvenes de la colonia no quieren realizar este tipo de trabajo.

En relación a los registros de observación se pudo identificar que dos de las viviendas donde habitan los beneficiarios de la pensión cuentan con antenas satelitales de TV, que funcionan con motores generados de energía eléctrica.

Además, la vestimenta utilizada por los beneficiarios al momento de realizar la entrevista, a simple vista se encuentra en buenas condiciones, aunque 8 de los 12 beneficiarios no utilizaban calzado.

Po otro lado, se identificó una población de control formada por adultos mayores de la comunidad Indígena La Promesa que no son beneficiarios del Programa de Pensión Alimentaria para Adultos Mayores en situación de pobreza y que de acuerdo a los datos recabados son 5 personas.

Las condiciones de las viviendas de los adultos mayores no beneficiarios de la pensión son similares a las de los beneficiarios de la pensión, los materiales utilizados y la forma de los ranchos no presentan diferencias.

Las cinco viviendas con adultos mayores que no son beneficiarios tienen en promedio 7 personas viviendo en el mismo hogar. No se observa en las viviendas antenas de TV satelital y la vestimenta de los adultos mayores está en peores condiciones que la de los beneficiarios de la pensión, según lo observado al momento de realizar la entrevista.

Los adultos mayores que no cobran la pensión, mencionaron que realizan recolección de miel de abeja en los meses de temporada (de noviembre a febrero) y posteriormente venden el producto en la comunidad indígena, lo que les genera un ingreso económico mínimo que va destinado a la compra de "provista" (alimentos). Además, mencionaron que ninguna persona que habita el hogar realiza algún tipo de trabajo remunerado fuera de la comunidad.

Mencionaron además que cuando tienen algún problema de salud, acuden al Puesto de Salud de la colonia. Nunca han salido de la comunidad por algún problema de salud por los costos que esto acarrearía.

Los adultos mayores no beneficiarios de la pensión mencionaron que cuando no tienen alimentos que consumir van a las viviendas de otros pobladores donde reciben alimentos al menos una vez al día, estas viviendas que comparten los alimentos son los hogares de los adultos mayores que reciben la pensión.

En la colonia Indígena La Promesa, la Secretaria de Emergencia Nacional realiza entrega de víveres dos a tres veces al año, generalmente en épocas de sequía o inundación, cada familia recibe 40 kilos de víveres en promedio en cada entrega, entre arroz, fideo, yerba, harina, azúcar, sal y galleta. Información dada por el líder comunitario, quien es el encargado de gestionar este tipo de ayuda.

Los adultos mayores de la población de control mencionaron que no acceden a la pensión ya que no cuentan con partida de nacimiento por lo tanto no tiene cedula de identidad policial. Tienen la esperanza de poder contar con la documentación requerida para de esa forma cobrar la pensión.

La posibilidad de acceder a alimentos de manera permanente, es la principal característica encontrada en los beneficiarios del Programa de Pensión Alimentaria para Adultos Mayores, 
que permite determinar un mejoramiento en la calidad de vida de los mismos, en comparación con la población de control, que no cuenta con alimentos en la vivienda de manera frecuente durante el mes.

\section{CONCLUSIONES}

Existe un mejoramiento de la calidad de vida de los beneficiarios del Programa de Pensión Alimentaria para Adultos Mayores en situación de pobreza en la Colonia Indígena La Promesa en el Departamento de Presidente Hayes, en lo relacionado a la posibilidad de acceder a una alimentación diaria.

No se observan otros aspectos que indiquen un mejoramiento en la calidad de vida de los beneficiarios del programa en relación a otras variables analizadas dentro de la investigación. La situación de extrema pobreza en la cual se encuentra la colonia Indígena La Promesa, hace que todo el núcleo familiar del beneficiario dependa del dinero recibido de la pensión, lo que no permite al beneficiario salir de la situación de extrema pobreza en la cual se encuentra.

En función a los resultados presentados, se identifican una serie de recomendaciones en torno a la aplicación de la presente ley en la comunidad Indígena La Promesa;

La pensión para adultos mayores en situación de pobreza, reduce la pobreza extrema (vinculado a alimentos) de los adultos mayores en la comunidad Indígena La Promesa; no así el índice de pobreza multidimensional, por lo tanto, es necesario replantear la Ley $\mathrm{N}^{\circ} 3728$ de forma a aumentar la eficiencia de la medida.

Se debería analizar la posibilidad de realizar los pagos de manera particular y en efectivo, en la propia comunidad, de modo a evitar la presencia de los intermediarios para el cobro.

Los valores que conservan las familias de la comunidad Indígena La Promesa, permiten desarrollar la ayuda mutua y la solidaridad entre ellas, compartiendo los alimentos en momentos de necesidad.

\section{REFERENCIAS BIBLIOGRÁFICAS}

Barrientos, A. (2003). Pensions and Developmet in South. The Geneva Papers on Risk and Insurance.

Barrientos, A. (2007). Nuevas estrategias de seguridad del ingreso en la vejez para los países de bajos ingresos. Asociación Internacional de la Seguridad Social. AISS.

Congreso Nacional. (1992). Constitución Nacional de la República del Paraguay. Congreso Nacional: Asunción.

Congreso Nacional. (2009). Ley № 3728 “Que establece el derecho a la pensión alimentaria para personas adultas mayores en situación de pobreza". Congreso Nacional: Asunción.

Dirección General de Estadística, Encuestas y Censos. (DGEEC). (2012). III Censo Nacional de Población y Viviendas para pueblos Indígenas. DGEEC: Asunción.

Zavattiero, C. (2010). Estimación del impacto de la ley de pensión alimentaria para personas adultas mayores en situación de pobreza en el Paraguay. III Taller: "Paraguay desde las ciencias sociales". Grupo de Estudios Sociales sobre Paraguay: Resistencia. 